This item was submitted to Loughborough's Research Repository by the author.

Items in Figshare are protected by copyright, with all rights reserved, unless otherwise indicated.

\title{
Assembling convolution neural networks for automatic viewing transformation
}

\section{PLEASE CITE THE PUBLISHED VERSION}

https://doi.org/10.1109/tii.2019.2940136

\section{PUBLISHER}

Institute of Electrical and Electronics Engineers (IEEE)

VERSION

AM (Accepted Manuscript)

\section{PUBLISHER STATEMENT}

Personal use of this material is permitted. Permission from IEEE must be obtained for all other uses, in any current or future media, including reprinting/republishing this material for advertising or promotional purposes, creating new collective works, for resale or redistribution to servers or lists, or reuse of any copyrighted component of this work in other works.

\section{LICENCE}

\section{All Rights Reserved}

\section{REPOSITORY RECORD}

Cai, Haibin, Lei Jiang, Bangli Liu, Yiqi Deng, and Qinggang Meng. 2019. "Assembling Convolution Neural Networks for Automatic Viewing Transformation". figshare. https://hdl.handle.net/2134/9912980.v1. 


\title{
Assembling Convolution Neural Networks for Automatic Viewing Transformation
}

\author{
Haibin Cai, Lei Jiang, Bangli Liu, Yiqi Deng, and Qinggang Meng, Senior Member, IEEE
}

\begin{abstract}
Images taken under different camera poses are rotated or distorted, which leads to poor perception experiences. This paper proposes a new framework to automatically transform the images to the conformable view setting by assembling different convolution neural networks. Specifically, a referential 3D ground plane is firstly derived from the RGB image and a novel projection mapping algorithm is developed to achieve automatic viewing transformation. Extensive experimental results demonstrate that the proposed method outperforms the state-ofthe-art vanishing points based methods by a large margin in terms of accuracy and robustness.
\end{abstract}

Index Terms-Automatic Viewing Transform, Convolution Neural Networks, Deep Learning.

\section{INTRODUCTION}

$\mathbf{H}$ UMAN has the capability of automatically transforming scenes observed with rotated viewing angles into a particular comfortable viewing setting, for example, the orientation of a door will always look like vertical under different head poses. However, most of the existing image sensors are based on the pinhole model [1] and lack the built-in viewing transformation ability. As a result, images taken under different viewing angles might have an uncomfortable viewing experience for human beings. Fig. 1 shows an example of two images taken under different viewing angles. To this end, automatic viewing transformation aims to transform an image taken under different viewing angle to a common horizontal viewing setting.

Automatic viewing transformation is useful in applications such as photography, mixture reality, online shopping, and human-computer interaction. For example, with this technology, image sensors will be able to improve the imaging quality by automatic compensating the impact caused by shaking or rotating. In mixture reality scenarios, an intelligent system is expected to automatically adjust the viewing angle of the scene according to the current pose of a subject. Via automatically transforming the living scenes taken inside a shopping market to a human-centered viewing setting, customers will have a better online shopping experience. Moreover, the transforming procedure can also serve as a preprocessing step to overcome the viewing angle challenges for research such as human action recognition [2], image understanding [3], and multisensory system integration [4].

This work was supported by YOBAN project(Newton Fund/Innovate UK, 102871), EPSRC CDT-EI and SukeIntel Co., Ltd.(Q. Meng is the corresponding author)

H. Cai, L. Jiang, B. Liu and Q. Meng are with the Computer Science, School of Science, Loughborough University, UK.

Y. Deng is with Hunan SukeIntel Co., Ltd, Changsha CEC Software Park, Changsha, Hunan, China.

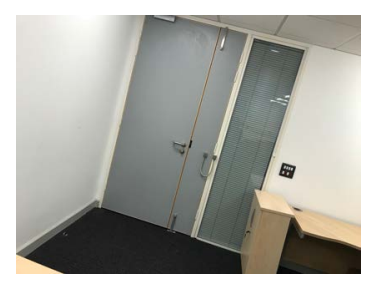

(a)

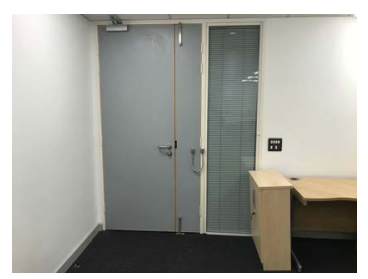

(b)
Fig. 1. Images taken under different viewing angles. (a) Rotated viewing angle. (b) Horizontal viewing angle. It can be observed that the left image is much harder for human to interpret than the right image due to the rotated viewing angle.

Existing viewing transformation methods are largely based on the calculation of vanishing points or line segments [510]. The cause of vanishing points is due to the perceptive projection, where parallel lines in 3D space will interact with each other at a point in the image plane. Using the geometric theory of the vanishing points, the corresponding transformation matrix can be modeled for viewing transformation. For example, Carroll et al. [10] proposed a nonlinear leastsquares optimization-based warping model which takes several user annotations including planar regions, straight lines, and associated vanishing points as constraints for viewing transformation. However, as mentioned by the authors, this method suffers from a complex user interface where a user has to understand the basic principles of perspective construction to be able to use it. Lee et al. [9] proposed an energy minimization framework to automatically correct the images via jointly modeling the camera parameters, vanishing points, and segments. The disadvantage of this type of methods is that they require high localization precision of the vanishing points, which is hard to achieve due to the uncertainty of the scene. In fact, the edges of some rotated objects might even lead to false detection of the vanishing points or lines.

Taking advantage of the outstanding interpretation ability of deep learning models, this paper proposes a new framework for automatic viewing transformation via assembling different convolution neural networks. More specifically, the Deep Ordinal Regression Network(DORN)[11] is employed to recover the depth information of the color image and the PSPNet[12] trained on the ADE20K[13] dataset is utilized to semantically segment different parts of the input image. Inspired by the feeling that human beings are good at using the ground plane in judging the direction of objects, this paper explores the possibility of using the ground plane as a reference to achieve automatic viewing transformation. We 
use the Ransac [14] algorithm to estimate the 3D ground plane and further propose a novel projection mapping algorithm to automatically transform the images to a conformable viewing setting. Thanks to the involvement of the $3 \mathrm{D}$ structures, the proposed method also has the capability to recover the scene at any viewing angles. Compared to the existing methods, the proposed method does not require the detection of vanishing points, thus makes it applicable in the scenarios where the detected vanishing points are insufficient or inaccurate. The contributions of this paper are listed as follows:

1. A new deep learning based framework is proposed for automatic viewing transformation. The framework seeks the possibility of using a referential plane for the warping of the image, which is fundamentally different from the existing vanishing point based methods.

2. A novel projection mapping algorithm is proposed to enable the system to be transformed to a conformable viewing setting.

3. A dataset is collected to evaluate the performance of existing methods. Experimental results show that the proposed method achieves great performance improvements over the state-of-the-art methods.

The rest of this paper is organized as follows. Section 2 reviews related work on image transformation. Section 3 presents the classic vanishing points based mapping method. The proposed method is described in detail in Section 4. Section 5 shows experimental evaluations with a variety of practical images. Finally, this paper is concluded with discussions in Section 6.

\section{RELATED WORK}

The cause of the uncomfortable viewing images is mainly due to the rotation of the image sensors. This phenomenon is well identified and many methods have been proposed to address this issue in the literature.

The geometric property of the vanishing points makes it an ideal solution for the viewing transformation. Gallagher et al. [15] used vanishing points to calculate the rotation towards the yaw angle of the camera and corrected the image via a simple back rotation of the image. Later, Chaudhury et al. [16] proposed a Ransac based approach to estimate two vanishing points and aligned the closer vanishing point with the Y-axis of the image via a post-multiplication operation. Santana et al. [17] utilized several long lines in the image to locate the vanishing points and performed image rectification based on a camera motion simulation. Lee et al. [6, 9] proposed an optimization framework which can simultaneously estimate the vanishing lines, vanishing points, and the camera parameters. Considering the difficulties and uncertainties in the accurate localization of the vanishing points, Carroll et al. [10] proposed a semiautomatic 2D image wrapping methods by asking the users to manually annotate several constraints such as planar regions of the scene, straight lines, and associated vanishing points. These constraints then serve as prior knowledge for the optimization of the image warping procedure. The main drawback of these methods is that they either require an accurate localization of vanishing points or an extra manual annotation operation.

There are some methods correcting the image by using the line segments [7, 18-20]. For example, He et al. [18] constructed an energy function to preserve the rotation of horizontal/vertical lines. By assuming that the users can give a rough rotation angle to create a perception rotation and the vertexes should be on the upright rectangular boundary of the output, they managed to construct the energy function and further solved it by using a two-stage optimation procedure. Observing that the straight line segments are not sufficient for panoramic images, Li et al. [19] improved the above method by further modeling the geodesic appearance of line segments into the energy function. Similarly, An et al. [7] parameterized the homography with camera parameters and designed a cost function to encode the measure of line segment alignment for image warping. Although an accurate result can be achieved by perfectly aligning the lines to a horizontal or vertical boundary, this type of methods suffer from the mis-detection of lines. This paper differs greatly from these methods in that a 3D wrapping solution is developed for the image transformation problem by using the estimated depth information.

\section{VAnishing Point BASEd Mapping Method}

The usage of vanishing points in correcting the image is a well-studied research topic [9, 15-17, 21, 22]. This section presents a Vanishing Point based Mapping method (VPM) for the calculation of the rotation matrix from three vanishing points. To ensure an accurate localization of the vanishing points, a manual procedure rather than automatical vanishing points detection algorithms $[8,17]$ is adopted.

Let $\alpha, \beta$, and $\gamma$ be the rotation angles towards the $X$ axis, the $Y$ axis, and the $Z$ axis respectively. Then, the rotation matrix can be formulated using Eq. 1. Assuming that the positions of the three localized vanishing points in the image coordinate system are $\left(x_{i}, y_{i}\right), i \in[0,2]$, they have the following relationship with their corresponding 3D locations $\left(X_{i}, Y_{i}, Z_{i}\right)$ in the world coordinate system according to the 3D projection rule [23]:

$$
\left\{\begin{array}{l}
x_{i}=f \frac{R_{00} X_{i}+R_{01} Y_{i}+R_{02} Z_{i}+t_{0}}{R_{20} X_{i}+R_{21} Y_{i}+R_{22} Z_{i}+t_{2}}+u_{0} \\
y_{i}=f \frac{R_{10} X_{i}+R_{11} Y_{i}+R_{12} Z_{i}+t_{1}}{R_{20} X_{i}+R_{21} Y_{i}+R_{22} Z_{i}+t_{2}}+v_{0} \\
i \in[0,2]
\end{array}\right.
$$

where $f$ is the focal length. $\left(u_{0}, v_{0}\right)$ is the coordinate of the principle point. The translation matrix is denoted as $\left(t_{0}, t_{1}, t_{2}\right)$. Considering that the vanishing point of axis $X$ has an infinite coordinate value of $X$, the value of $\left(x_{0}, y_{0}\right)$ can then be approximating to $\left(f \frac{R_{00}}{R_{20}}+u_{0}, f \frac{R_{10}}{R_{20}}+v_{0}\right)$. The same rule

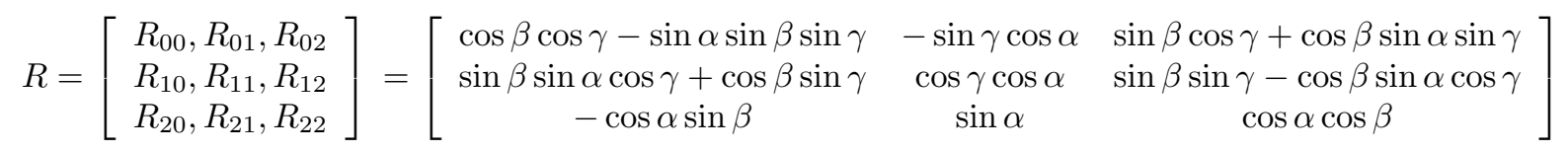




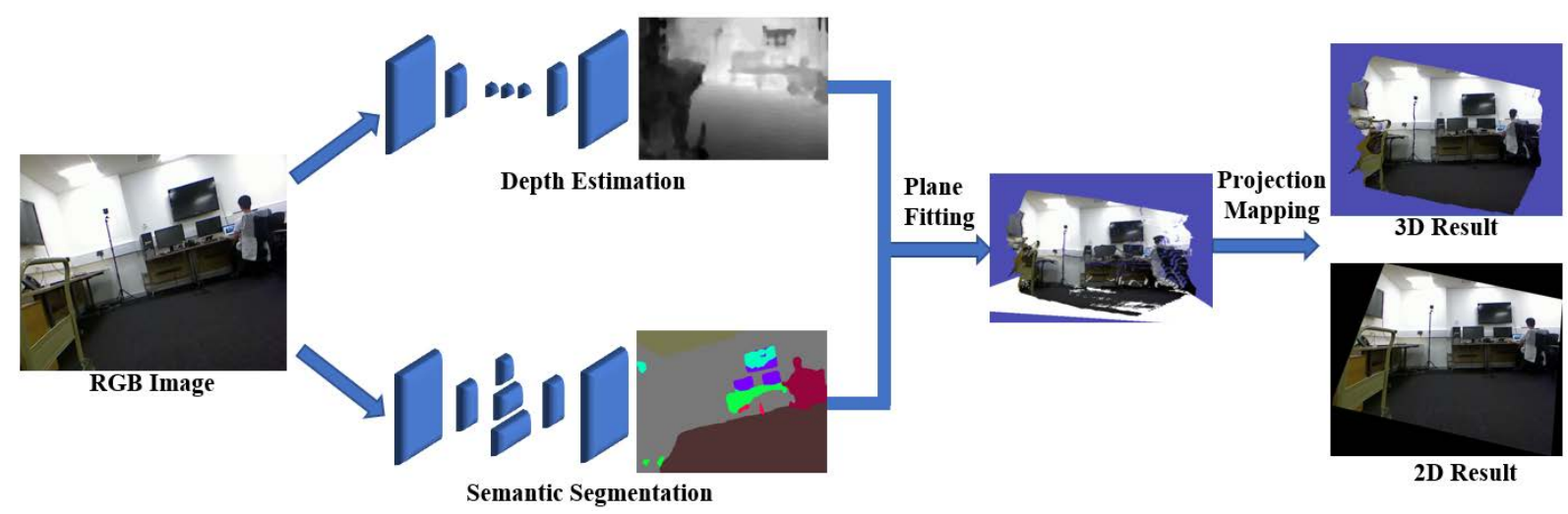

Fig. 2. The framework of the proposed method. The image after the plane fitting operations shows the reconstructed 3D point clouds. The white plane in this image is the fitted plane. The output of this framework can be both 3D point clouds and a transformed 2D image.

applies to the other two vanishing points. Thus, Eq. 2 can be simplified into the following equation.

$$
\left\{\begin{array}{l}
x_{i} \approx f \frac{R_{0 i}}{R_{2 i}}+u_{0} \\
y_{i} \approx f \frac{R_{1 i}}{R_{2 i}}+v_{0} \\
i \in[0,2]
\end{array}\right.
$$

Combining Eq. 1 and Eq. 3, the following equation can be formed to solve the rotation matrix:

$$
\left\{\begin{array}{l}
\frac{y_{1}-y_{0}}{x_{1}-x_{0}}=\frac{\cos \gamma \sin \beta+\sin \gamma \cos \beta \sin \alpha}{\cos \gamma \cos \beta \sin \alpha-\sin \gamma \sin \beta} \\
\frac{y_{2}-y_{1}}{x_{2}-x_{1}}=\frac{\sin \gamma \sin \beta \sin \alpha-\cos \gamma \cos \beta}{\cos \gamma \sin \beta \sin \alpha+\sin \gamma \cos \beta} \\
\frac{y_{0}-y_{2}}{x_{0}-x_{2}}=\frac{-\sin \gamma}{\cos \gamma}
\end{array}\right.
$$

Once the rotation matrix is determined, the image transformation can be performed using the following equation [5]:

$$
P^{\prime}=K(K R)^{-1} P
$$

where $P$ and $P^{\prime}$ represent for the position of an pixel in the original image and transformed image respectively. $K$ is the intrinsic parameter of the image sensor.

\section{Proposed Method}

The proposed method mainly consists of the DRON for depth estimation, the PSPNet for image segmentation, a Ransac based method for ground plane estimation, and a novel projection mapping method for the viewing transformation. The following subsections describe the detail of these methods.

\section{A. Framework Description}

Fig. 2 shows the framework of the proposed method. The input of the framework is a RGB image which can be captured via a variety of sensors such as a webcam, a Kinect sensor, or a GoPro camera. The aim is to achieve automatic coordinate transformation via the frequently occurred ground plane information. To this end, the DRON and the PSPNet are used for depth estimation and semantic segmentation, respectively. The $3 \mathrm{D}$ point clouds of the ground plane can be obtained by fusing the outputs from these two CNNs. Although it is also possible to extract the 3D ground plane directly from the estimated depth map, the combination of two methods can produce a more accurate 3D ground plane fitting result. Then, a Ransac based algorithm is used to estimate the accurate plane surface whose normal vector is selected for the calculation of the rotation matrix. Finally, a novel $3 \mathrm{D}$ projection mapping procedure is designed to achieve an automatic viewing transformation.

\section{B. Depth Estimation}

Estimating depth information from a single RGB image is an ill-posed problem. Recently, significant improvements have been achieved with the help of deep convolutional neural networks, indicating that it is possible to apply the estimated depth information to tasks such as $3 \mathrm{D}$ reconstruction and scene understanding. In this paper, DORN [11] is employed for depth estimation due to its advantages in both high accuracy and fast processing speed. Inside the DORN, a full-image encoder is designed and a spacing-increasing discretization strategy is developed to recast the depth network learning as an ordinary regression problem. The network is implemented on the Caffe [24] platform and trained on the NYU Depth Dataset V2 dataset [25].

\section{Semantic Segmentation}

Semantic segmentation aims to recognize object categories in an image in the pixel level. By replacing the fully-connected layer with a convolution layer, the Fully Convolutional Networks (FCN) [26] has demonstrated the effectiveness of deep neural networks in semantic segmentation, inspiring many novel networks been proposed [27]. This paper adopts the PSPNet [12] due to its good performance in segmenting the ground plane. The main novelty of this network lies in the design of the pyramid pooling module, which empirically proves to be essential in improving the segmentation accuracy. The model is trained on the ADE20K dataset [13], which contains more than $20 \mathrm{~K}$ images with dense annotations and wide distribution of scenes.

\section{Ground Plane Fitting}

The output of the depth estimation and semantic segmentation images are combined to reconstruct the $3 \mathrm{D}$ point clouds 
of the ground plane. It should be noted that there is abnormal data in both the depth image and the segmented map, which leads to noises in the recovered 3D points clouds. To reduce the effect of these noises, the Ransac algorithm is used to efficiently and robustly fit the ground plane. More specifically, it randomly selects three points to compute the hypothesis plane since three points determine a plane. A point is judged as an inlier if its distance to the hypothesis is smaller than a predefined threshold. In practical, the threshold value of 600 $\mathrm{mm}$ is found to be robust for the plane detection task. After repeating this process several times, many hypotheses can be generated. Then, the hypothesis with the most inner points is selected. The final plane is determined via a least square plane fitting over these inner points.

\section{E. Projection Mapping}

Once the ground plane is fitted, the viewing transformation can be accomplished via the proposed projection mapping method. Firstly, the 3D point clouds of the scene can be recovered using the following equation:

$$
\left\{\begin{array}{l}
X=\frac{u-u_{0}}{f_{x}} * Z \\
Y=\frac{v-v_{0}}{f_{y}} * Z
\end{array}\right.
$$

where $(u, v)$ is the location of a pixel in the RGB image. $\left(u_{0}, v_{0}, f_{x}, f_{y}\right)$ stands for the intrinsic parameters of the camera and $(X, Y, Z)$ is the position of the reconstructed 3D point.

Given the normal vector of the ground plane $N=$ $\left(n_{x}, n_{y}, n_{z}\right)$ and the target transformation vector $V=$ $\left(v_{x}, v_{y}, v_{z}\right)$, the rotation matrix can be determined using the Rodrigues' rotation formula which is defined as follows:

$$
\left\{\begin{array}{l}
\theta=\arccos \left(\frac{N * V}{|N| *|V|}\right) \\
{\left[\begin{array}{c}
c_{x} \\
c_{y} \\
c_{z}
\end{array}\right]=\left[\begin{array}{c}
n_{y} * v_{z}-n_{z} * v_{y} \\
n_{z} * v_{x}-n_{x} * v_{z} \\
n_{x} * v_{y}-n_{y} * v_{x}
\end{array}\right]} \\
K=\left[\begin{array}{c}
0,-c_{z}, c_{y} \\
c_{z}, 0,-c_{x} \\
-c_{y}, c_{x}, 0
\end{array}\right] \\
R=\cos (\theta) I+\sin (\theta) K+(1-\cos (\theta)) K^{2}
\end{array}\right.
$$

where $\theta$ is the angle between the normal vector and transformation vector. Their cross product vector $\left(c_{x}, c_{y}, c_{z}\right)$ is used as the rotation axis. $K$ and $R$ are the cross product matrix and rotation matrix, respectively. Considering that human tend to use the ground plane as a reference in the perception system and the power of gravity makes objects pointing towards the ground plane, the target transformation vector $V$ is set to be $(0,1,0)$ to create the comfortable viewing setting.

Using the calculated rotation matrix, the reconstructed 3D point clouds can be rotated and projected to a specific viewing angle and position through the following equation:

$$
\left\{\begin{array}{l}
P^{\prime}=R * P+T \\
u^{\prime}=u_{0}+f_{x} * \frac{X^{\prime}}{Z^{\prime}} \\
v^{\prime}=v_{0}+f_{y} * \frac{Y^{\prime}}{Z^{\prime}} \\
R=\left[\begin{array}{c}
R_{0}, R_{1}, R_{2} \\
R_{3}, R_{4}, R_{5} \\
R_{6}, R_{7}, R_{8}
\end{array}\right]
\end{array}\right.
$$

where $R$ is the rotation matrix to transfer the points from the camera coordinate system to the world coordinate system. $T$ is a user-specified transition matrix to set the location of the camera. $P^{\prime}$ is the position of a transferred 3D Point $\left(X^{\prime}, Y^{\prime}, Z^{\prime}\right)$ in the world coordinate system. $P$ stands for the original 3D point $(X, Y, Z)$ in the camera's coordinate system. $\left(u^{\prime}, v^{\prime}\right)$ stands for the projection position of the point $P^{\prime}$ in the transformed image.

By letting the transition matrix $T$ be $(0,0,0)$ and combining Eq. 6 and Eq. 8, the projection mapping function can be simplified as follows:

$$
\left\{\begin{array}{l}
u^{\prime}=u_{0}+\frac{R_{0} *\left(u-u_{0}\right) * f_{x} * f_{y}+R_{1} *\left(v-v_{0}\right) * f_{x}^{2}+R_{2} * f_{x}^{2} * f_{y}}{R_{6} *\left(u-u_{0}\right) * f_{y}+R_{7} *\left(v-v_{0}\right) * f_{x}+R_{8} * f_{x} * f_{y}} \\
v^{\prime}=v_{0}+\frac{R_{3} *\left(u-u_{0}\right) * f_{y}^{2}+R_{4} *\left(v-v_{0}\right) * f_{x} * f_{y}+R_{5} * f_{x} * f_{y}^{2}}{R_{6} *\left(u-u_{0}\right) * f_{y}+R_{7} *\left(v-v_{0}\right) * f_{x}+R_{8} * f_{x} * f_{y}}
\end{array}\right.
$$

After feeding the rotation matrix $R$ calculated in Eq. 7 to Eq. 9, the transformed image can be obtained. It should be noted that although the system is designed to transform the scene into the ground plane guided viewing setting, it can also perform a transformation to any viewing angle settings via giving a specific rotation and transition matrix.

The main novelty of the projection mapping lies in the derivation of the transformation matrix using the ground plane information and the applying of the derived matrix for the automatic viewing transformation. The scene observed under different viewing angles often has different contents, which will probably result in some black holes or pixels in the transformed image. To deal with these holes and create a smooth image, the bilinear interpolation filter is used in the final 2D image.

\section{EXPERIMENTS}

The algorithms are implemented on a computer with an Intel Core i7-7700K CPU and a GTX 1080Ti GPU. Each algorithm has been run for over 1000 times and their average processing time is shown in Table 1 . The resolution of the estimated depth map from DORN is fixed at $353 * 257$ and further resized to $640 * 480$, which is the resolution of the RGB image. During the testing, we use the same set of model and camera parameters for all the images. It can be seen from the table that the large computation cost mainly lies in the depth estimation and semantic segmentation. Although the 3D plane-fitting algorithm and the projection mapping algorithm are conducted only on the CPU, they are able to achieve realtime performance.

TABLE I

TIME PERformance OF THE PROPOSED METHOD

\begin{tabular}{|c||c|}
\hline Algorithm & Time (s) \\
\hline Depth Estimation & 2.86 \\
Semantic Segmentation & 2.15 \\
3D Plane Fitting & 0.023 \\
Projection Mapping & 0.0034 \\
\hline
\end{tabular}

\section{A. Perspective Transformation Dataset}

To evaluate the performance of the proposed method, the Perspective Transformation Dataset (PTD) which contains 280 


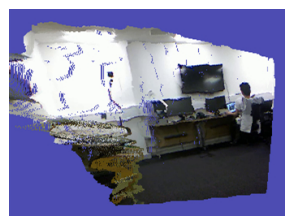

(a)

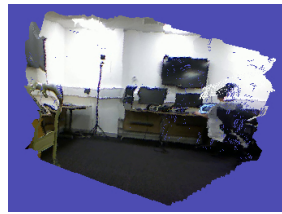

(d)

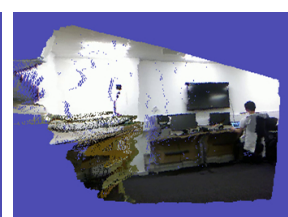

(b)

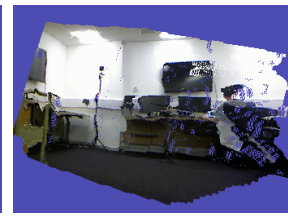

(e)

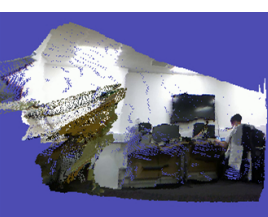

(c)

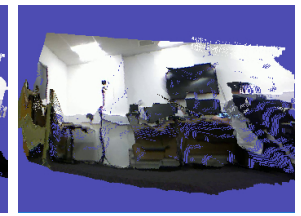

(f)
Fig. 3. Viewing transformation results with specific conditions. The transition matrix is set to be $(0,0,3000) \mathrm{mm}$ to demonstrate the effect of changing in distances. The $\beta$ of the first row images and second row images are set to be $30^{\circ}$ and $-30^{\circ}$ respectively. From the first column to the third column, the $\alpha$ is increased equally from $-20^{\circ}$ to $20^{\circ}$. The $\gamma$ is set to be $0^{\circ}$ in these images to outline the affect of the former setting.

images captured under 10 different indoor scenes is collected. It employs three different webcams, a Kinect sensor, and a GoPro sensor to ensure the diversity in the hardware sensors. Among them, the GoPro sensor has a wider viewing angle than the other sensors, which results in some distortions in the captured images. During the capturing process, the sensors are randomly rotated to a certain angle (ranges from $0^{0}$ to $30^{\circ}$ ) to make the images various in rotations.

\section{B. Accuracy Measurement}

The transformation results are usually measured by conducting a user study to judge the effectiveness of the methods or simply looking at the transformed images [6, 9, 16, 17]. Apart from directly comparing the transformed results, this paper further performs a quantitative comparison by measuring the angle difference of a specific line that should be horizontal after the transformation. The specific line can be determined by manually selecting two points inside the image. Then, the angle error can be determined using the following equation:

$$
e=\operatorname{atan}\left(\frac{y_{1}-y_{2}}{x_{1}-x_{2}}\right)
$$

where $e$ stands for the angle difference. $\left(x_{1}, y_{1}\right)$ and $\left(x_{2}, y_{2}\right)$ are the coordinate of two manually selected points on the line that should be horizontal in the transformed image.

\section{Performance of $3 D$ map}

The proposed method has an advantage over the 2D image wrapping algorithms in that it can perform a transformation to any viewing settings given a target rotation matrix and transition matrix. Fig. 3 presents some snapshots of the 3D map under specific viewing condition. Looking at the images in the same row, we can easily find out the influence of the $\alpha$ angle. The effect of changing in the $\beta$ angle can be found by comparing images in each column.

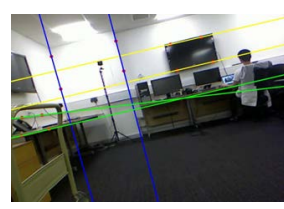

(a)

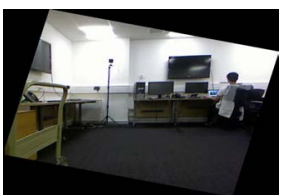

(b)

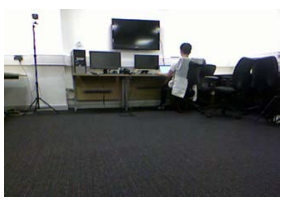

(c)
Fig. 4. Illustrations for the VPM method. (a) The way to retrieve the vanishing points via manually clicking points and calculating the intersection point of the selected lines. (b) The transformed results using the accurately calculated vanishing points and the theory presented in Section III. (c) An example of the images where the third vanishing point cannot be determined.

\section{Performance of the VPM method}

This subsection presents the performance of the VPM method in transforming images. Due to the projection, the parallel lines in the $3 \mathrm{D}$ world will intersect at a point in the image. Thus, three different axis directions will result in three corresponding vanishing points. Fig. 4a shows the manual procedure to localize the three vanishing points whose position can be used to calculate the rotation matrix according to Eq. 4 . The transformed result is shown in Fig. 4b. As pointed out by many researchers, some photos do not have enough content for the localization of three vanishing points [6, 17], which limits the application of the VPM method. Fig. $4 \mathrm{c}$ shows one of these images where the third vanishing point cannot be localized.

\section{E. Comparison to the State-of-the-Art}

This subsection compares the transformation results of the proposed method with the state-of-the-art methods. Although some related work $[7,17,20,22]$ has been proposed recently, none of these papers have open sourced their implementation, which causes the difficulty in further comparisons. To boost further research, the binary version of this software will be made publicly available.

Fig. 5 shows the performance of the methods with images taken under different angles and illuminations. The original images are shown in the first column. The results of the proposed method and Google's method [16] are positioned in the second column and third column receptively. Although both methods achieve satisfactory results in these circumstances, the details from the enlarged TV object show that the proposed method outperforms Google's method in most of the cases. For example, in the first row of Fig. 5, the boundary of the TV in the middle column looks like a rhombus while it is a rectangle in the third column, showing that the transformation result of the proposed method is closer to the realistic situation.

Fig. 6 compares the viewing transformation results of the two methods with images captured by the GoPro camera. Due to the wide angle lens, images taken by this sensor have some distortions which might lead to unstable performance for Google's method. The first two rows demonstrate that both methods can accurately transform the images in some circumstances. While images in the last five rows show that the transformation result of the proposed method is more stable and accurate.

Fig. 7 presents the transformation results of the two methods under some other scenes with different angles. These images 

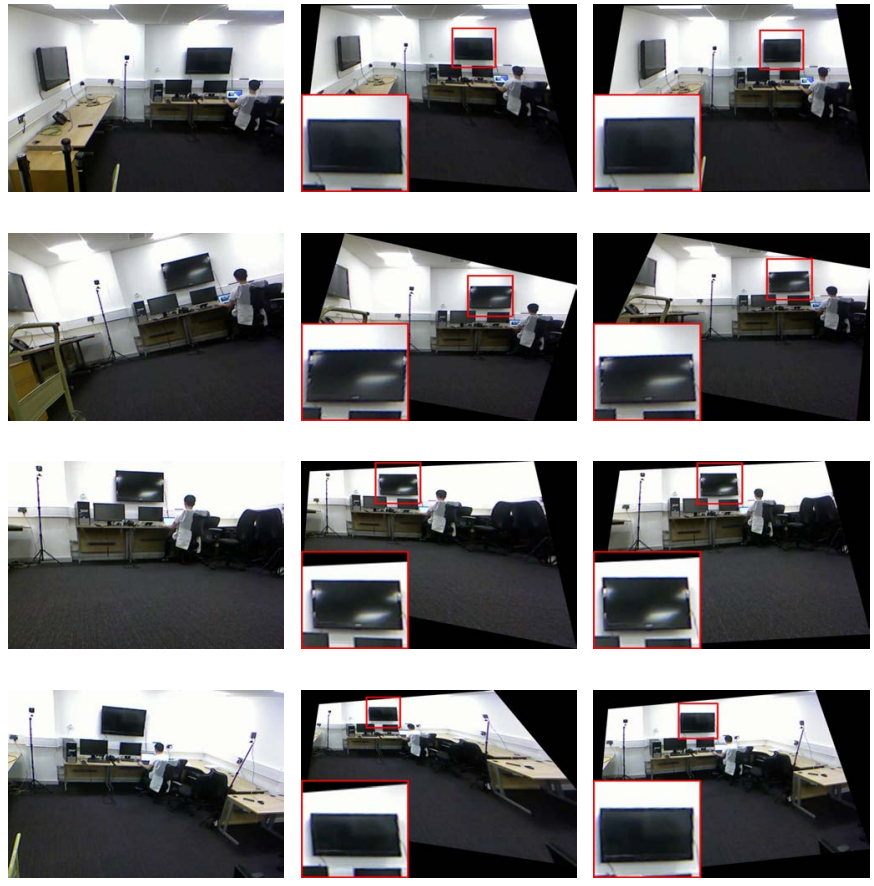

Original

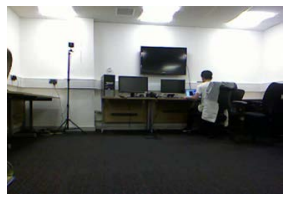

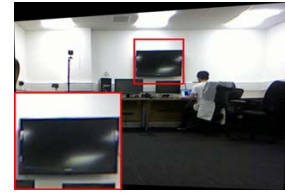

Google [16]

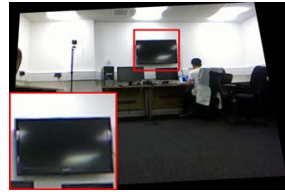

Proposed Method
Fig. 5. Comparison of viewing transformation results with images captured under different angles and illuminations. The TV object in the second column and third column are enlarged to facilitate reading. The enlarged parts are marked with red rectangles.

are captured by normal webcams and the Kinect RGB sensor. The images are not cropped to give an overall view of the transformation results. It can be seen from the second row of the figure that [16] sometimes fails to transform the images even when they are not distorted. The cause of this might due to the wrongly detected vanishing points. Though the proposed method achieves accurate and robust performance over [16] in most of the cases, it relies on the reconstruction of the 3D ground plane, which might hinder its broad application for the images where the ground information is not presented.

Fig. 8 shows the accuracy of the three methods using the measurement presented in Section V-B. The red line and blue line indicate the accuracy of the proposed method and [16] receptively. As can be seen from the figure, the performance of the VPM method (green line) is much inferior to the other two methods. The cause of this phenomenon is either due to the missing of the third vanishing point or the inaccurately localized vanishing points. Note that the precise position of the vanishing points is extremely difficult to achieve when the lines are nearly parallel in the image.

Table II shows a quantitative comparison of the accuracy of the three methods in the PTD database. The proposed method and [16] both achieve around $57.1 \%$ under the condition that the tolerance is within $2^{\circ}$. When the tolerance is bigger than
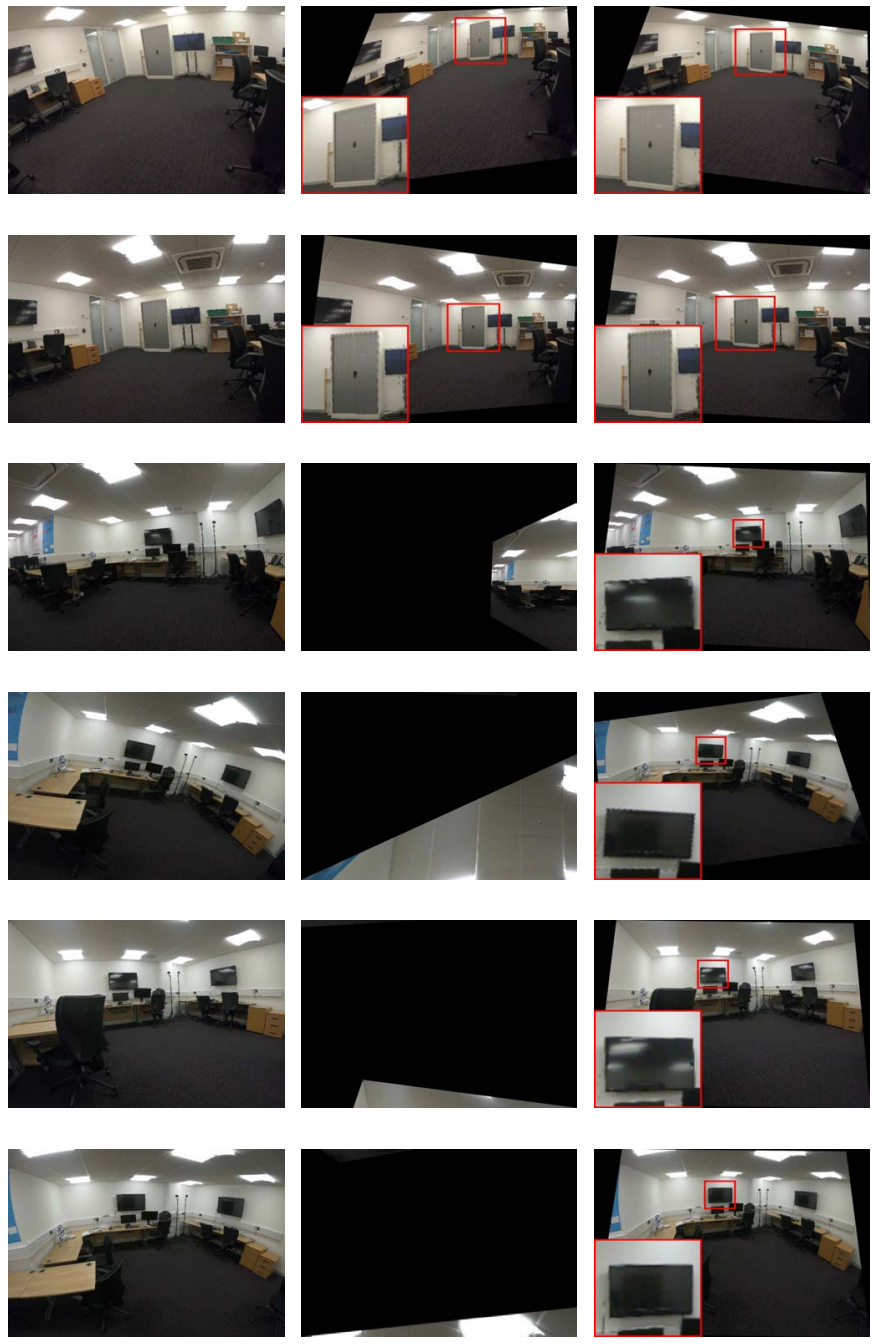

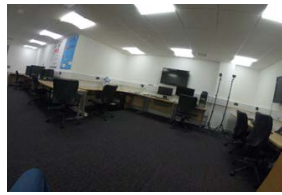

Original

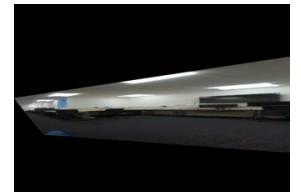

Google [16]

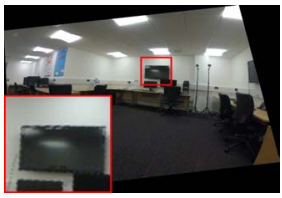

Proposed Method
Fig. 6. Comparison of viewing transformation results with images captured using the GoPro camera with a wide angle lens.

$2^{\circ}$, the proposed method outperforms [16] by a large extent. For instance, over $30 \%$ improvements have been achieved by the proposed method when the tolerance is within $6^{\circ}$.

TABLE II

QUANTITATIVE COMPARISON OF THE ANGLE ERROR OF THE THREE METHODS IN THE COLLECTED DATABASE.

\begin{tabular}{|c||ccc|}
\hline Method & $e \leq 2^{\circ}$ & $e \leq 4^{\circ}$ & $e \leq 6^{\circ}$ \\
\hline VPM & $21.4 \%$ & $42.9 \%$ & $46.4 \%$ \\
Google [16] & $57.1 \%$ & $60.7 \%$ & $64.3 \%$ \\
The proposed method & $57.1 \%$ & $75.0 \%$ & $96.4 \%$ \\
\hline
\end{tabular}

\section{CONCLUSION}

This paper achieves a significant improvement in automatical viewing transformation, not only does it get rid of the 

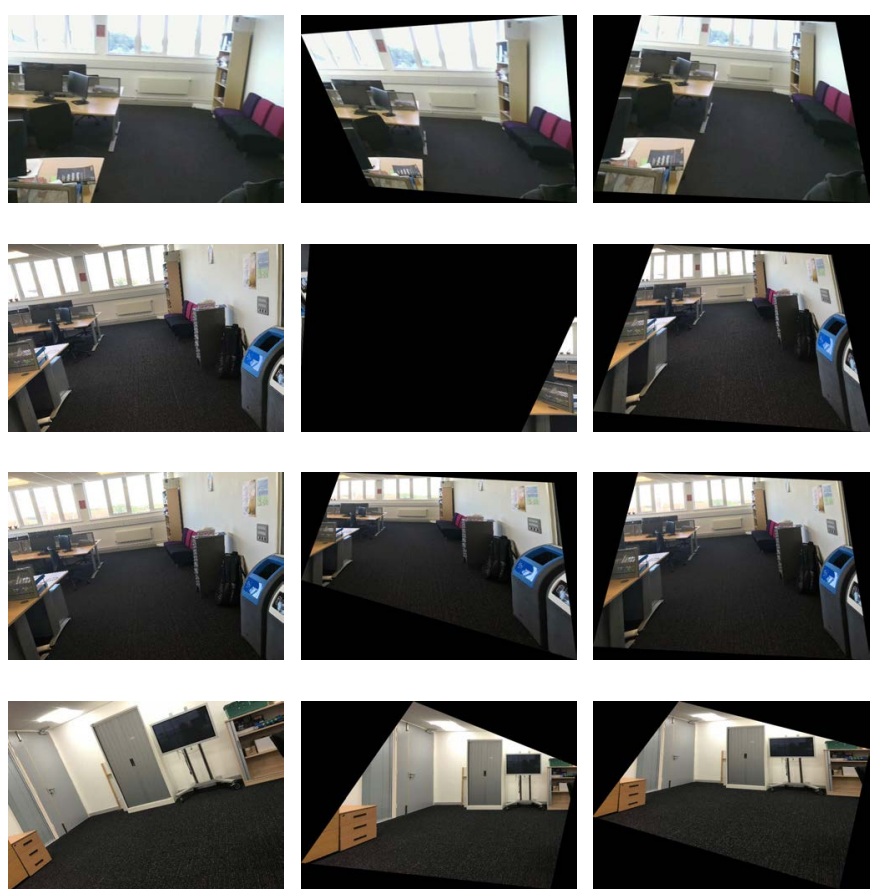

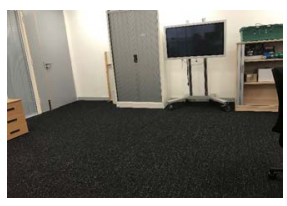

Original

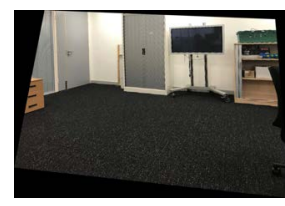

Google [16]

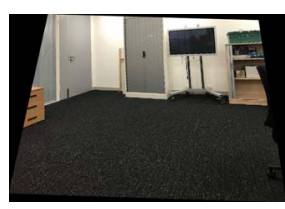

Proposed Method
Fig. 7. Comparison of viewing transformation results with images captured under some other scenes with different angles.

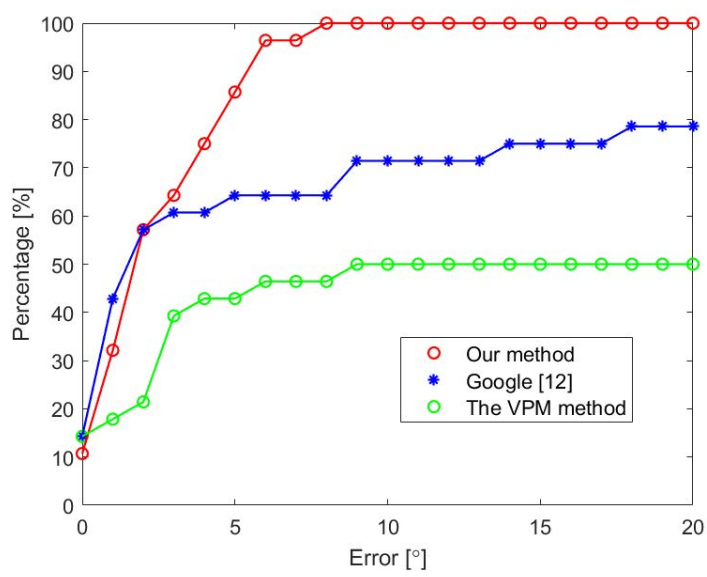

Fig. 8. The accuracy of the three methods on the PTD database. The $\mathrm{x}$ axis means angle tolerance. The y axis represents for the percentage of the images whose angle error is smaller than certain tolerance. general requirement of detecting vanishing points or lines, but it also outperforms the state-of-the-art methods in terms of accuracy and robustness. Based on the assumption that the ground plane can be observed in the captured images, a deep learning based viewing transformation framework and a novel projection mapping algorithm are designed to adjust the perspective. Experimental evaluations have demonstrated that the proposed algorithm can perform automatic viewing transformation for images taken under different positions and rotations, paving the way for video analysis applications such as mixture reality, human behavior recognition, and calibration-free multi-camera system integration.

Future works have been targeted as follows: 1) Collecting and annotating a large perspective transformation dataset via synthesizing images using 3D models of the scene. 2) Exploring the possibility of using an end-to-end network for the viewing transformation task. 3) Designing intelligent multicamera human-machine interaction applications based on the proposed techniques.

\section{REFERENCES}

[1] J. Heikkilä, "Geometric Camera Calibration Using Circular Control Points," IEEE Trans. Pattern Anal. Mach. Intell., vol. 22, no. 10, pp. 1066-1077, 2000.

[2] Y. Guo, Y. Li, and Z. Shao, "On Multiscale SelfSimilarities Description for," IEEE Trans. Ind. Informat., vol. 13, no. 6, pp. 3017-3026, 2017.

[3] H. Liu, S. Chen, and N. Kubota, "Intelligent video systems and analytics: A survey," IEEE Trans. Ind. Informat., vol. 9, no. 3, pp. 1222-1233, 2013.

[4] J. Chen, K. Li, Q. Deng, K. Li, and P. S. Yu, "Distributed Deep Learning Model for Intelligent Video Surveillance Systems with Edge Computing," IEEE Trans. Ind. Informat., pp. 1-1, 2019.

[5] R. Hartley and A. Zisserman, "Multiple view geometry in computer vision," Cambridge University Press, 2003.

[6] H. Lee, E. Shechtman, J. Wang, and S. Lee, "Automatic upright adjustment of photographs," in Proc. IEEE Conf. Comput. Vis. Pattern Recog., 2012, pp. 877-884.

[7] J. An, H. I. Koo, and N. I. Cho, "Rectification of planar targets using line segments," Mach. Vis. Appl., vol. 28, no. 1-2, pp. 91-100, 2017.

[8] F. M. Mirzaei and S. I. Roumeliotis, "Optimal estimation of vanishing points in a Manhattan world," in Proc. IEEE Int. Conf. Computer Vision, 2011, pp. 2454-2461.

[9] H. Lee, E. Shechtman, J. Wang, and S. Lee, "Automatic Upright Adjustment of Photographs With Robust Camera Calibration," IEEE Trans. Pattern Anal. Mach. Intell., vol. 36, no. 5, pp. 833-844, 2014.

[10] R. Carroll, A. Agarwala, and M. Agrawala, "Image warps for artistic perspective manipulation," ACM Trans. Graphics, vol. 29, no. 4, p. 127, 2010.

[11] H. Fu, M. Gong, C. Wang, K. Batmanghelich, and D. Tao, "Deep Ordinal Regression Network for Monocular Depth Estimation," in Proc. IEEE Conf. Comput. Vis. Pattern Recog., 2018, pp. 2002-2011. 
[12] H. Zhao, J. Shi, X. Qi, X. Wang, and J. Jia, "Pyramid scene parsing network," in Proc. IEEE Conf. Comput. Vis. Pattern Recog., 2017, pp. 6230-6239.

[13] B. Zhou, H. Zhao, X. Puig, T. Xiao, S. Fidler, A. Barriuso, and A. Torralba, "Semantic Understanding of Scenes Through the ADE20K Dataset," Int. J. Comput. Vis., vol. 127, no. 3, pp. 302-321, 2019.

[14] M. A. Fischler and R. C. Bolles, "Random sample consensus. A paradigm for model fitting with apphcahons to image analysm and automated cartography," Graphics and Image Processing, vol. 24, no. 6, pp. 381-395, 1981.

[15] A. C. Gallagher, "Using Vanishing Points To Correct Camera Rotation In Images," in Proc. Conf. Comput. Robot Vis., no. 2, 2005, pp. 460-467.

[16] K. Chaudhury, S. DiVerdi, and S. Ioffe, "Autorectification of user photos," Proc. Int. Conf. Image Processing, pp. 3479-3483, 2014.

[17] D. Santana-Cedrés, L. Gomez, M. Alemán-Flores, A. Salgado, J. Esclarín, L. Mazorra, and L. Alvarez, "Automatic correction of perspective and optical distortions," Comput. Vis. Image Understanding, vol. 161, pp. 1-10, 2017.

[18] K. He, H. Chang, and J. Sun, "Content-aware rotation," in Proc. IEEE Int. Conf. Comput. Vis., 2013, pp. 553560.

[19] D. Li, K. He, J. Sun, and K. Zhou, "A geodesicpreserving method for image warping," in Proc. IEEE Conf. Comput. Vis. Pattern Recog., 2015, pp. 213-221.

[20] J. Jung, B. Kim, J. Y. Lee, B. Kim, and S. Lee, "Robust upright adjustment of 360 spherical panoramas," Visual Computer, vol. 33, no. 6-8, pp. 737-747, 2017.

[21] C. Hughes, P. Denny, M. Glavin, and E. Jones, "Equidistant fish-eye calibration and rectification by vanishing point extraction," IEEE Trans. Pattern Anal. Mach. Intell., vol. 32, no. 12, pp. 2289-2296, 2010.

[22] B. Kim, J.-Y. Lee, J. Jung, and G. Miller, "Automatic orientation adjustment of spherical panorama digital images," U.S. Patent Application, vol. 10, no. 127, p. 637, 2018.

[23] R. M. Haralick, "Using perspective transformations in scene analysis," Comput. Graphics and Image Processing, vol. 13, no. 3, pp. 191-221, 1980.

[24] Y. Jia, E. Shelhamer, J. Donahue, S. Karayev, J. Long, R. Girshick, S. Guadarrama, and T. Darrell, "Caffe: Convolutional Architecture for Fast Feature Embedding," in Proc. ACM Int.Conf. Multimedia, 2014, pp. 675-678.

[25] N. Silberman, D. Hoiem, P. Kohli, and R. Fergus, "Indoor segmentation and support inference from rgbd images," in Proc. Eur. Conf. Comput. Vis., 2012, pp. 746-760.

[26] E. Shelhamer, J. Long, and T. Darrell, "Fully convolutional networks for semantic segmentation," in Proc. IEEE Conf. Comput. Vis. Pattern Recog., 2014, pp. 34313440.

[27] L.-C. Chen, G. Papandreou, I. Kokkinos, K. Murphy, and A. L. Yuille, "DeepLab: Semantic Image Segmentation with Deep Convolutional Nets, Atrous Convolution, and Fully Connected CRFs." IEEE Trans. Pattern Anal. Mach. Intell., vol. 40, no. 4, pp. 834-848, 2018.

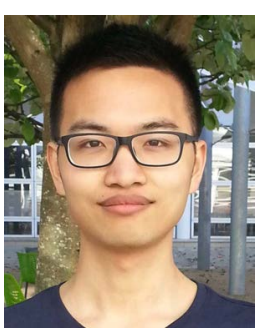

Haibin Cai received the M.S. degree in computer science from the Zhejiang University of Technology, Hangzhou, China, in 2015, and the Ph.D. degree in computer science from the University of Portsmouth, Portsmouth, U.K., in 2018. He is currently a Lecturer in Computer Science, Loughborough University. His research interests include gaze estimation, motion recognition, facial expression recognition, object tracking, image processing, and machine learning.

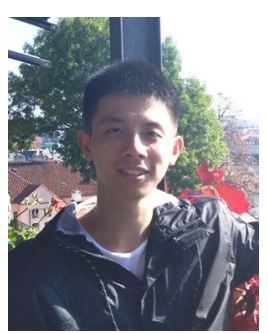

Jiang Lei received the M.S. degree in computer science from the Loughborough University, UK in 2018. He is currently pursuing the Ph.D. degree in computer science, Loughborough University. He is interested in image processing, object tracking, simultaneous localization and mapping, robotics, sematic segmentation, human behaviour analysis, and deep learning.

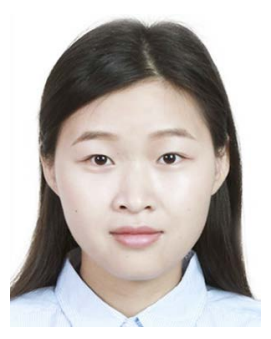

Bangli Liu received the B.Eng. degree at the China Jiliang University in 2012 and the M.Sc. degree at East China University of Science and Technology in 2015, and the Ph.D. degree in computer science at the University of Portsmouth, UK, in 2018. She is currently a Research Associate in Loughborough University. Her research interests include computer vision, pattern recognition, machine learning, image super-resolution, human motion analysis, defect detection.

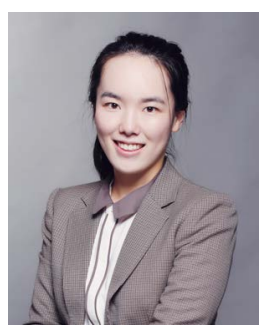

Yiqi Deng received the B.Sc. degree in Applied Mathematics from the National University of Defence Technology, China in 2011 and the Ph.D. degree in computer science from the University of College London, U.K., in 2018. She is interested in image processing, biological computing, and deep learning.

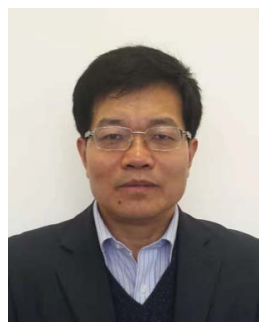

Qinggang Meng (M'06-SM'18) received the BSc and MSc degrees from the School of Electronic Information Engineering, Tianjin University, China, and the Ph.D. degree in computer science from Aberystwyth University, U.K. He is currently a Professor in Robotics and AI with the Department of Computer Science, Loughborough University, UK. He is a fellow of the Higher Education Academy, UK. His research interests include biologically inspired learning algorithms and developmental robotics, service robotics, agricultural robotics, robot learning and adaptation, multi-UAV cooperation, human motion analysis and activity recognition, pattern recognition, artificial intelligence, computer vision, and embedded intelligence. 